\title{
Theoretical Foundation for Jung's "Mandala Symbolism" Based on Discrete Chaotic Dynamics of Interacting Neurons
}

\author{
V. GONTAR \\ International Group for Chaos Studies at Ben-Gurion University of the Negev, P.O. Box 653,84105, Beer-Sheva, Israel
}

(Received 22 October 1998)

\begin{abstract}
Based on discrete chaotic dynamics algorithms different patterns in a form of mandalas have been generated. This fact gives us the possibility to make a link between mechanism of biochemical reaction dynamics undergoing in brain resulted to the brain creativity process in form of mandalas. Obtained patterns can be related to the space distributed chemicals according to the law of extended principle of maximum entropy, consideration of the information exchange during biochemical transformations, mass conservation law and discrete chaotic dynamics principles.
\end{abstract}

Keywords: Mandala, Chaos, Artificial brain, Discrete dynamics

\section{INTRODUCTION}

Discrete chaotic dynamics is a new theory specially developed for the complex and living systems behavior. This theory, based on a new concept of time and space using calculus of discrete difference equations versus continuous differential equations [1]. Application of this theory to the modeling of complex biochemical reaction dynamics demonstrated, among the other results, time series and patterns similar to EEG, ECG and mandalas.

Based on this fact we are going to propose theoretical explanation for the possible mechanism of the brain creativity in a form of mandalas. According to Jung [2], "mandala as psychological phenomena appear spontaneously in dreams, in certain states of conflict, and in cases of schizophrenia. Very frequently they contain a quaternity or a multiple of four, in a form of a cross, a star, a square, an octagon, etc".

"The fact that images (mandalas) of this kind have under certain circumstances a considerable therapeutic effect on their authors is empirically proved and also readily understandable, in that they often represent very bold attempts to see and put together apparently irreconcilable opposites and bridge over apparently hopeless splits. Even the mere attempt in this direction usually has a healing effect, but only when it is done spontaneously. Nothing can be expected from an artificial repetition or a deliberate imitation of such images." [2]. 
If we could succeed in understanding deeply the underlying mechanisms of biochemical reactions related to our brain activity then we can use modern computer technologies to help people in producing their own "mandalas" for practical use.

Speaking about living systems dynamics we need to emphasize the difference between non-living and living systems behavior. For the time being all existing laws of nature do not make difference between these two classes of objects. From Lotka, who found mathematical model for chemical oscillatory behavior and Schredinger with his claim to use equations obtained in physics for biological systems modeling up to nowadays there are no other ideas which could introduce the specificity of living systems in basic equation and therefore find adequate formalism.

What is the specificity of living systems? First of all is their ability of information storing and exchange in addition to the energy, pulses and mass exchange. The second is their ability to the self-reproduction through soft, not elastic interactions. These features should be included in the initial theory which is pretended on the description and mathematical modeling of living systems behavior as main principles. To construct such a theory we have chosen the chemical reaction formalism, which in a formal way reflects the fact of appearance of a new element from initial components or just transformation of one element to another.

$\mathrm{A}+\mathrm{B}=\mathrm{C}$ in a formal way says that for example living cell $\mathrm{A}$ interacts with living cell $\mathrm{B}$ and this interaction reduces a new cell $\mathrm{C}$. This formal notation includes in consideration the fact of birth of a new element. To bring to this ability of the living systems an information exchange we suppose that all chemicals or elements of the systems involved in the chemical transformations can affect the rates of the interactions. To denote these influences of constituents we just put the arrows going from A, B, C on characteristics of the process:

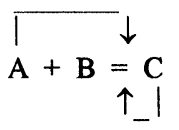

Following this approach we can increase the complexity of the consideration - number of elements and types of reactions - for any systems with practically unlimited numbers of elements with all possible interconnections on masses exchange and "information exchange" levels.

The next step is to write the equations reflecting proposed type of interaction. Classical physics and chemistry at that point applying to the theory of collisions (elastic) and theory of probability to connect the rate of chemical reaction. These equations known as kinetic mass action law, also named as rate or balance equations. No new physical principles reflecting the specificity of the living systems mentioned above are presented in this law and it is hard to expect that using them we can succeed in finding adequate results for living systems. Our goal in this paper is to construct new basic equations for living systems dynamics with well defined meaning of the parameter and principles.

\section{BACKGROUND}

Breaking results, related to the living systems dynamics, have been obtained in chaos theory, when it became clear that chaotic regimes and "chaotic systems" have not only the same right to exist in nature as the systems with regular deterministic behavior, but in fact extremely sensitive to the infinitely small changes of the initial conditions or parameters. The last is very much similar to what we call "information exchange" in living systems when infinitely small amount of information/from the energetic point of view and measure/can drastically affect and change the living systems behavior. To use chaotic systems as a model for the living systems dynamics looking attractive from that point of view, because giving us the formal way to include in consideration the important role of information for living system behavior.

The chaotic regimes as it is well known can be generated by computer's simulation of the systems of differential equations. To do this one thing is still claiming for explanation: how is it possible to use 
continuous differential equations, when by definition of chaotic regimes infinitely small changes in initial conditions or parameters drastically changing solutions in spite of principle of continuity, when solutions should converge? How theory of perturbation can coexist with the principle of the absence of small parameters in chaotic systems?

All these problems can be solved and new equations for living systems dynamics will be derived by introducing in consideration a new calculus calculus of iterations. To come to this point we need to start with chemical thermodynamics, where the principle of maximum entropy (minimum of free energy) is used for deriving algebraic equations of thermodynamics mass action law:

$$
\prod_{i=1}^{N} X_{i}^{\nu_{l i}}=K_{l}
$$

$K_{l}$ - constant of equilibrium for $l$-reaction. $X_{i}-$ concentrations of " $i$ " element, $\nu_{l i}$ - stoichiometric coefficients for any mechanisms of chemical transformation

$$
\begin{array}{ll}
\sum_{i=1}^{N} \nu_{l i} A_{i}=0 & \begin{array}{l}
i=1,2, \ldots, N \\
l
\end{array}=1,2, \ldots, N-M .
\end{array}
$$

Added by the mass conservation law equations:

$$
\sum_{i=1}^{N} a_{i j} X_{i}=b_{j} \quad j=1,2, \ldots, M,
$$

we will complete the system of algebraic equation in the case of equilibrium. Here $a_{i j}$ - "molecular" matrix, defined the number of component of type " $j$ " in $i$ th element, $b_{j}$ - initial concentration of $j$ th component.

As it is well known thermodynamics is not analyzing the dynamical changes in time of constituents, but giving us equations for calculation of final distribution of elements composing systems (just one point in concentration space when $t \rightarrow \infty$ ). The next step in constructing adequate calculus to the living systems dynamics mathematical modeling is to refer to the theory of dimensionality $[1,3]$.
It happens that invariant of the theory of dimensionality we have the same mathematical forms as equilibrium constants. That analogy opens a possibility to make a link between principle of maximum entropy and general postulates of the theory of dimensionality and expend principle of maximum entropy on all states of multicomponent systems even far from equilibrium. That will bring us to the systems of algebraic equations for describing kinetics and dynamics of chemical transformations [4]. And the last step to the calculus of the difference equations is to include in the basic algebraic equations dependence for parameters from concentrations calculated in previous moments. The type of these dependence reflect the "information exchange" we are including in consideration. Following [5] we need to substitute $K_{l}$ by in [1] by $\pi_{l}$

$$
\begin{aligned}
\pi_{l}\left(X\left(t_{q}\right), r\right)= & \tilde{K}_{l} \exp \left\{-\left[\sum_{i=1}^{N} \alpha_{l i} X_{i}\left(t_{\mathrm{q}-s}\right)\right.\right. \\
& \left.\left.+\sum_{i=1}^{N} \beta_{l i} X_{i}(r \otimes)\right] / t_{\mathrm{c}}\right\},
\end{aligned}
$$

where $\alpha_{l i}, \beta_{l i}$ are empirical parameters, characterizing the intensities of the feedbacks influences in time $-\alpha_{l i}$, and $\beta_{l i}$ - influences of the space distributed concentrations of neighbors $X_{i}(r \otimes)$ - on $l$ th chemical reaction, $\tilde{K}_{l}$ - empirical parameters, characterizing the steady states.

\section{MATHEMATICAL MODELING OF THE BRAIN CREATIVITY PROCESSES}

We are considering brain as interconnected neurons. Each neuron presents itself as a "living system" with the ability of information exchange with all others connected with it. Operation of each neuron will be modeled as individual "biochemical reactor". Internal parameters responsible for the intracellular reaction dynamics have also dependence on conditions connected to this neuron and other neurons (Fig. 1). Finally we have complex interconnected network of neurons which are operating according to the initial hypothesis of the 


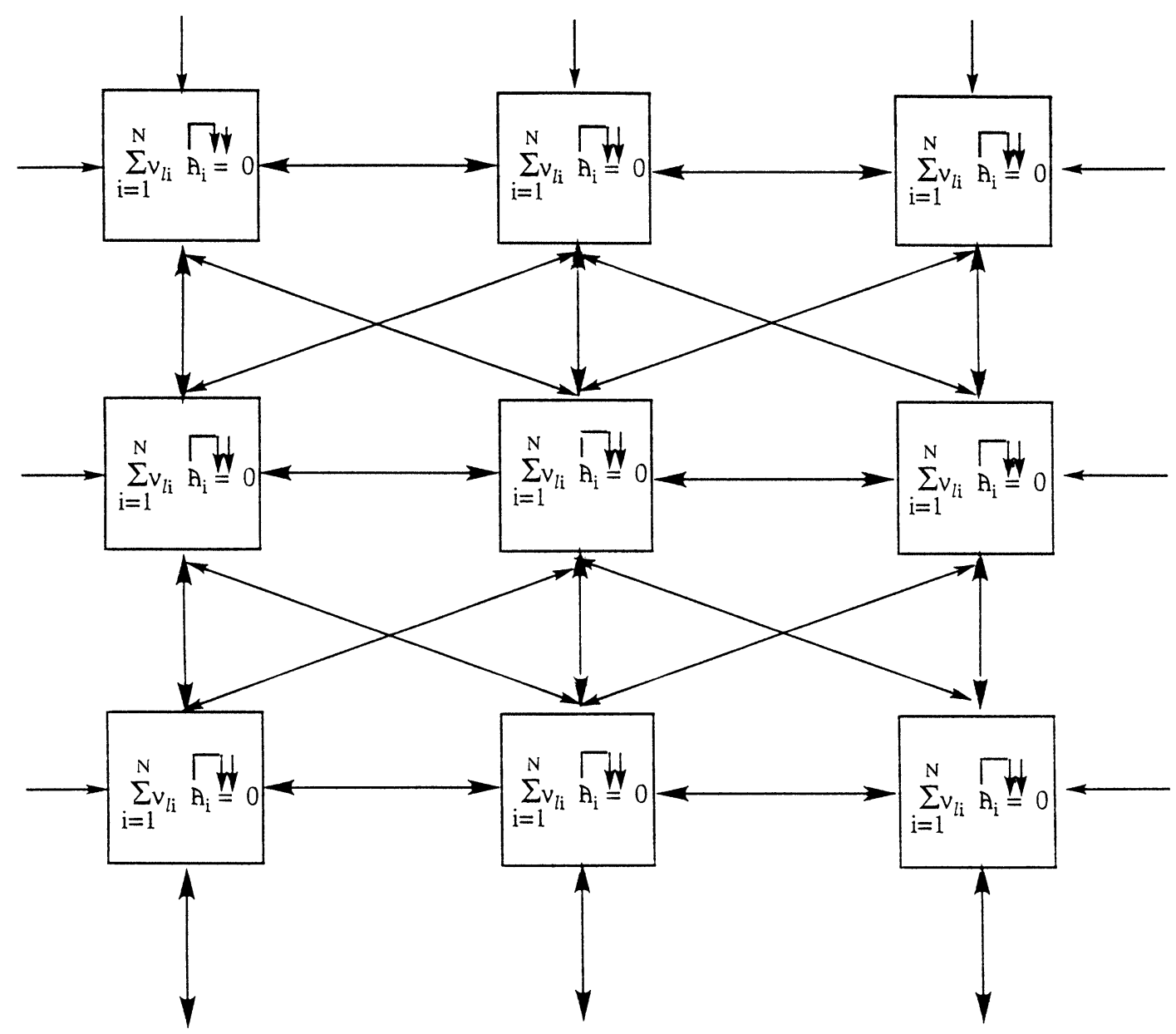

FIGURE 1 Schematic presentation of interacting neurons modeled in terms of biochemical reactions mechanism.

mechanism of biochemical reactions in the individual neuron and information exchange between the neurons. All systems operate according to the discrete dynamics principles based on the extension of maximum entropy principle, mass conservation law, theory of dimensionality and new basic equations. Using the laws of nature for mathematical modeling of brain activity should have advantages compared to the neural networks, cellular automata and other computer methods, used for the same purpose because the general physical principles will constrain us from meaningless solutions and will direct to the real mathematical solutions close to the reality.
In that respect we are coming to the conclusion that use of difference equations not only frees from logical contradictions between discrete and continuous time and space, chaotic and deterministic paradigms, but also much more simpler from the computational point of view, which is extremely important for practical purpose for such a level of computational complexity.

As a result of the computations we will get the evolution of space distributed concentrations of chemicals in each neuron. It is reasonable to suppose that these concentrations patterning is the basis for our internal brain creativity process. The fact that the patterns calculated by our model 
similar to the mandalas appeared in the human cultures of Indians, Tibetans, Europeans can be taken as some kind of confirmation that all of them have scientific background related to the biochemical transformations of chemicals in our brain, according to the modern discrete chaotic dynamics laws.

Standing in the beginning of these studies we need to realize great potential, coming from the detailed understanding of creative brain operations and we hope to present further results in this fascinating field.

In Figs. 2-6 you can see the results of the calculations performed for two-dimensional $(160 * 160$ pixels) square lattice, where each cells of the lattice is interconnected with close neighborhood. The color of each pixel corresponds to the amount of

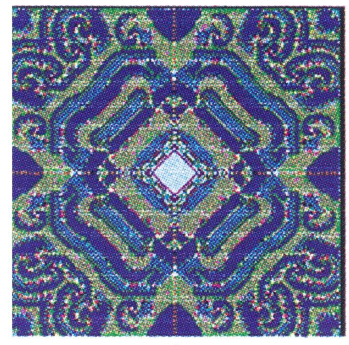

1

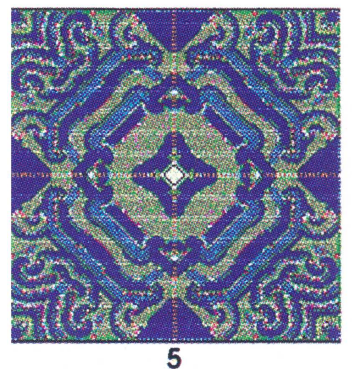

5

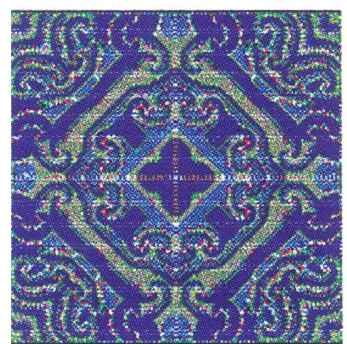

9

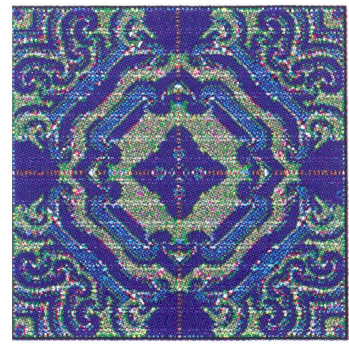

13

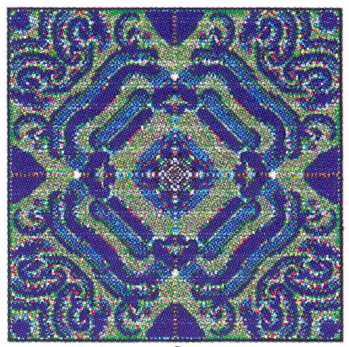

2

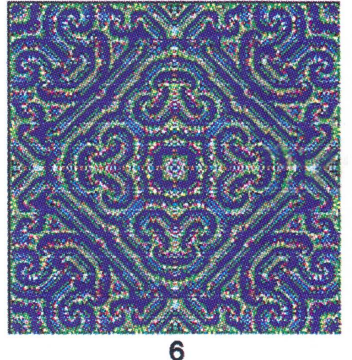

6

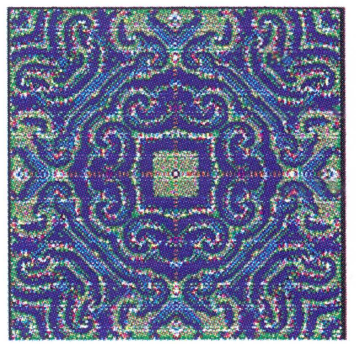

10

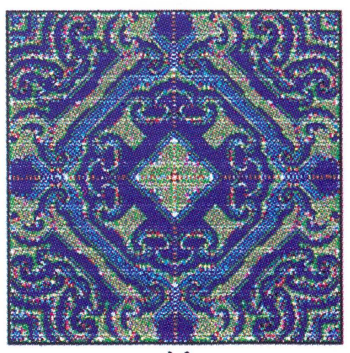

14

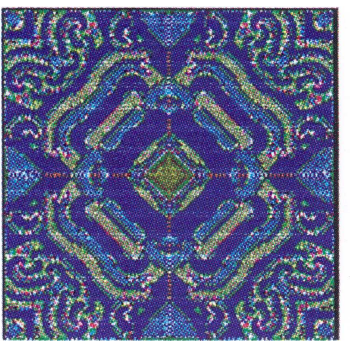

3
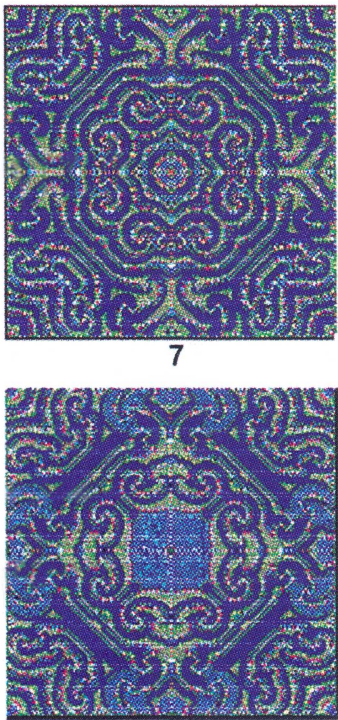

11

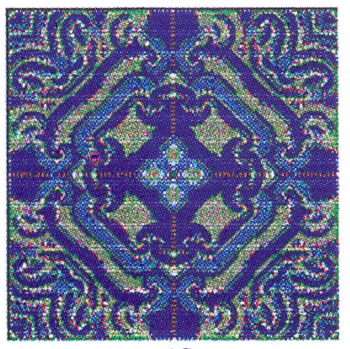

15

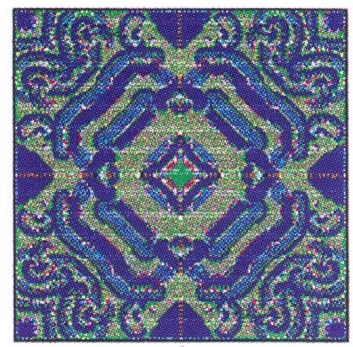

4
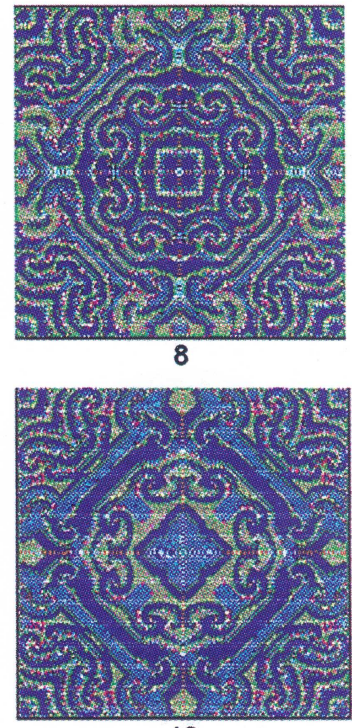

12

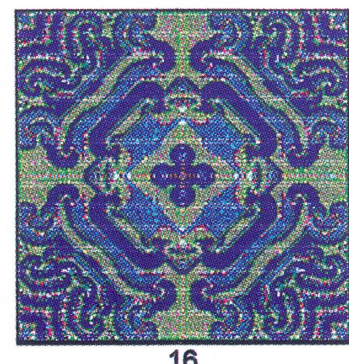

16

FIGURE 2 Mandalas calculated by the discrete dynamics algorithms. (See Color Plate I.) 


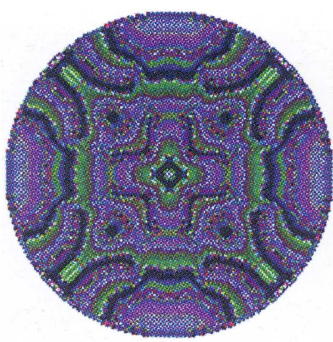

1

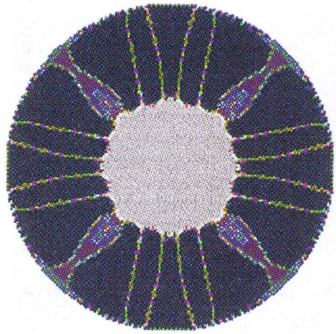

5

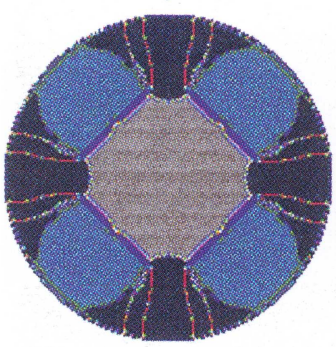

9

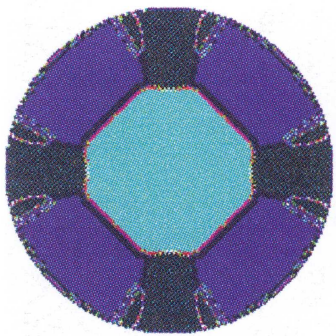

13

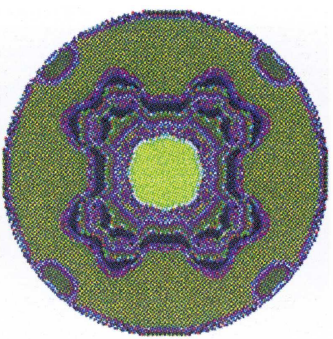

2

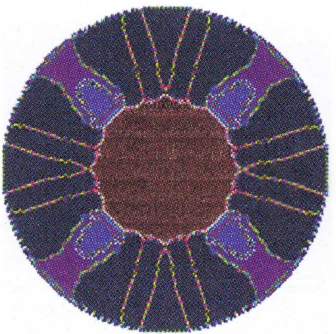

6

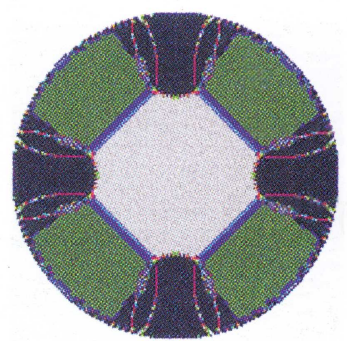

10

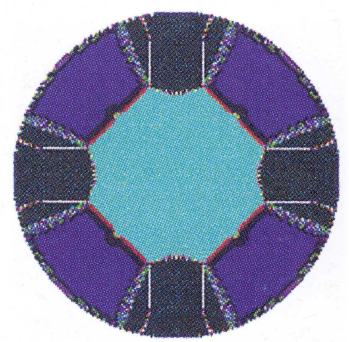

14

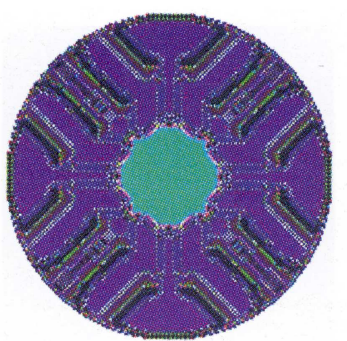

3

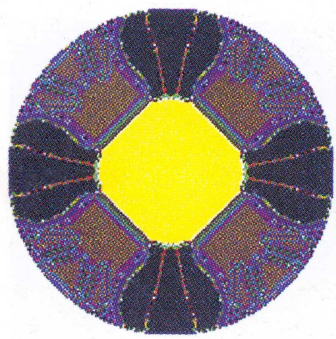

7

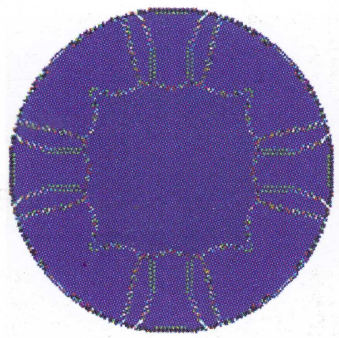

11

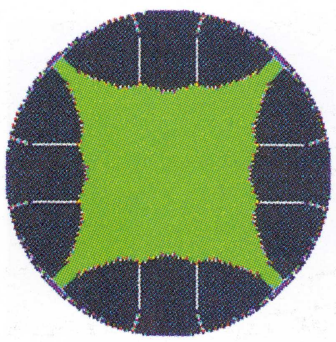

15

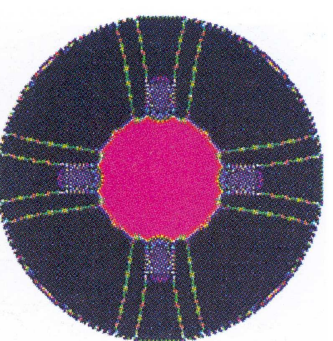

4

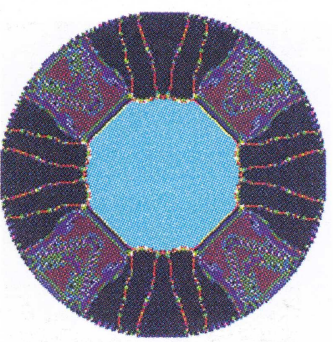

8

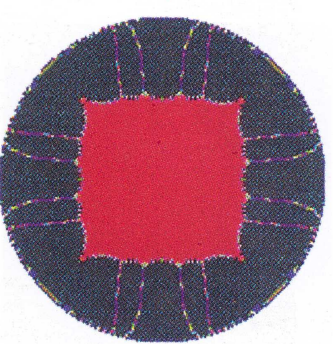

12

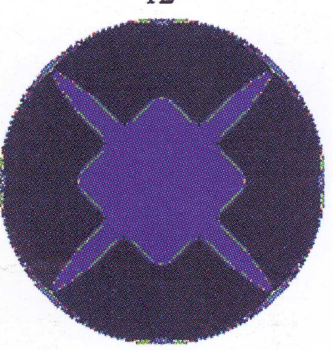

16

FIGURE 3 Mandalas calculated by the discrete chaotic dynamics algorithms. (See Color Plate II.)

the concentration of one of the chosen constituent. The mechanisms of biochemical transformation we have used for generation of the presented mandalas:

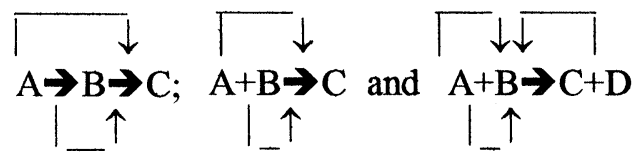

\section{CONCLUSIONS}

The proposed theoretical approach is based on using first physical principles for biochemical reactions dynamics. Corresponding mathematical model $(1,3,4)$, derived from these principles, reflects the natural constraints for biochemical reactions dynamics and therefore should have a 

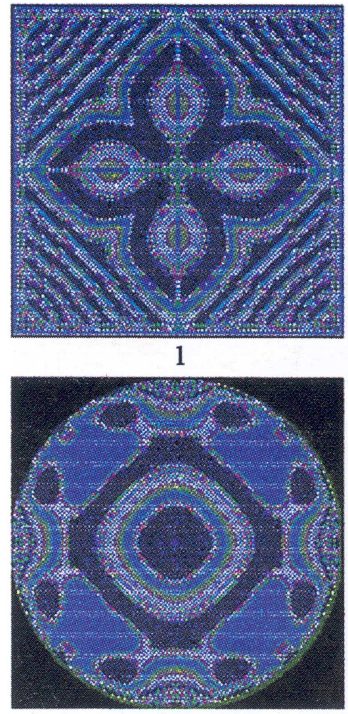

5

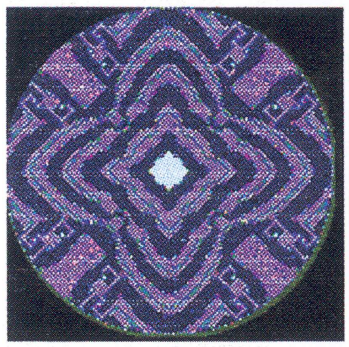

9

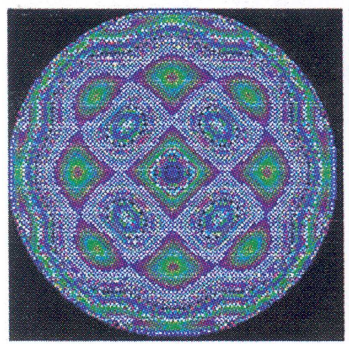

13

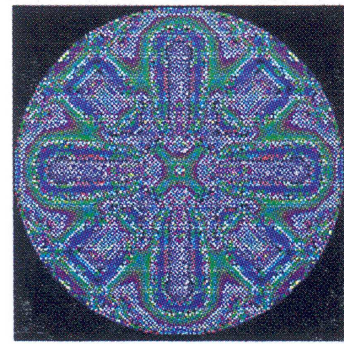

17
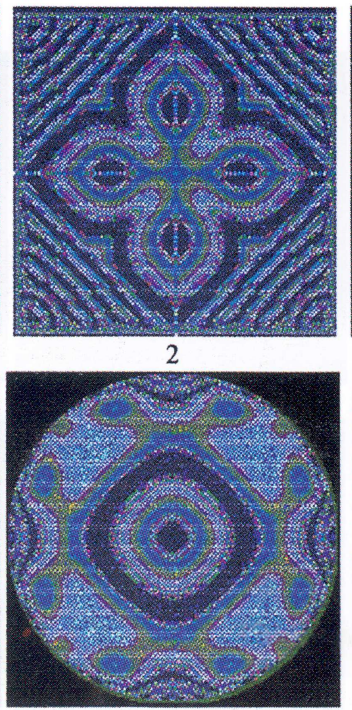

6

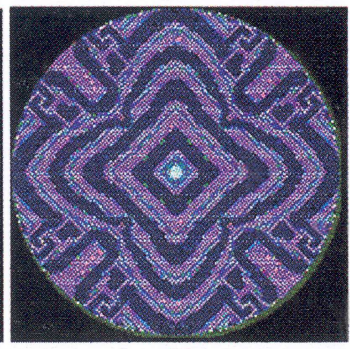

10

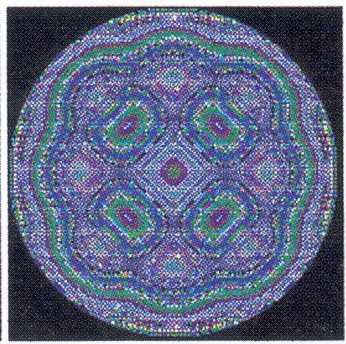

14

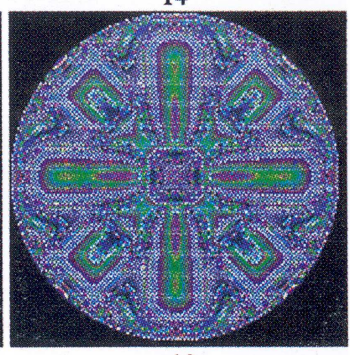

18
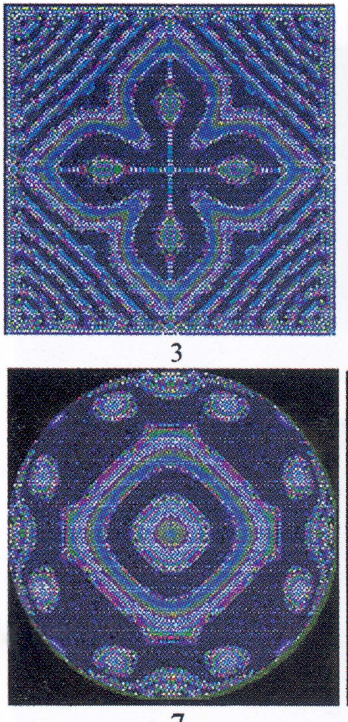

7

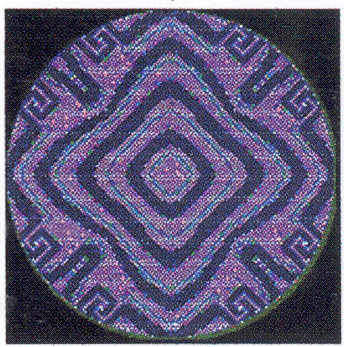

11

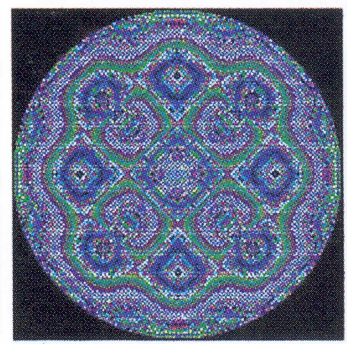

15

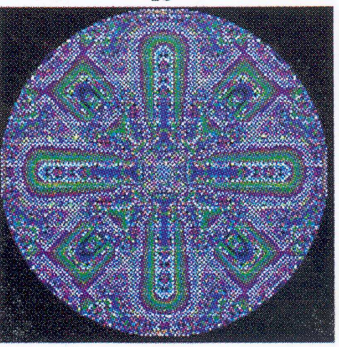

19

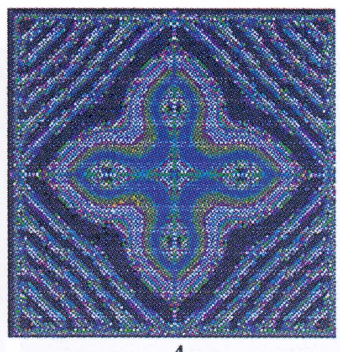

4

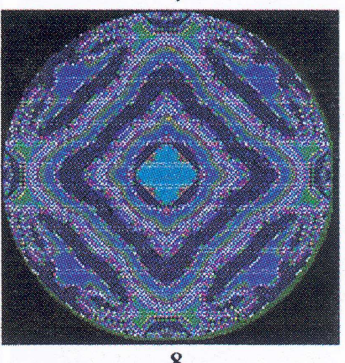

8

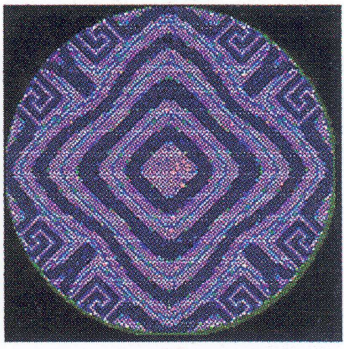

12

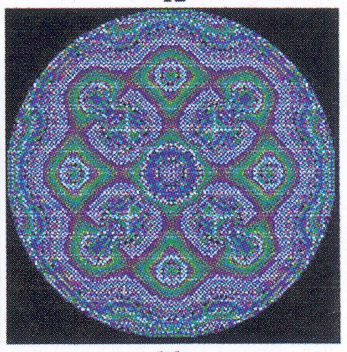

16

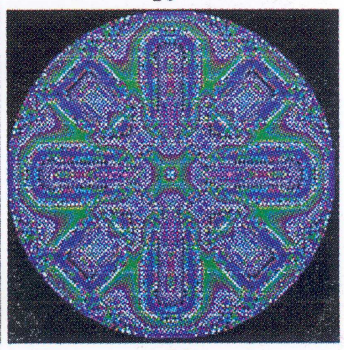

20

FIGURE 4 Mandalas calculated by the discrete dynamics algorithms. (See Color Plate III.) 

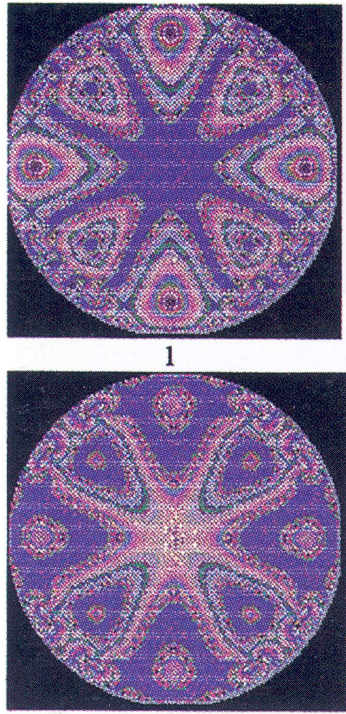

5

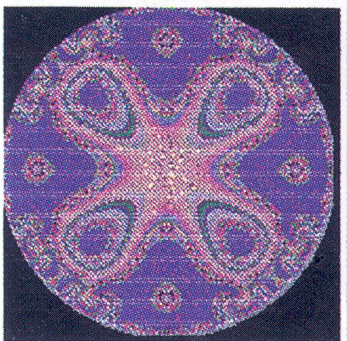

9

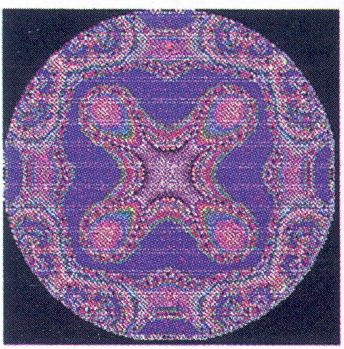

13

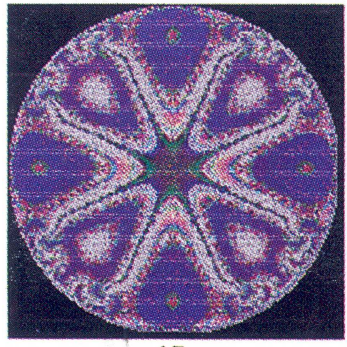

17

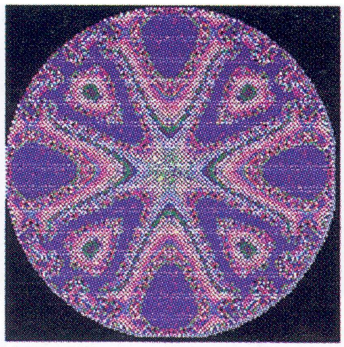

2

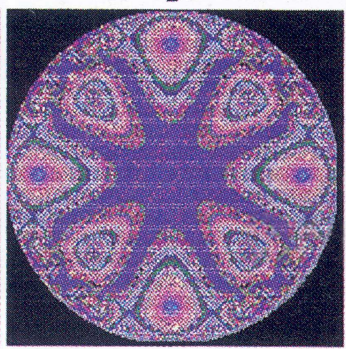

6

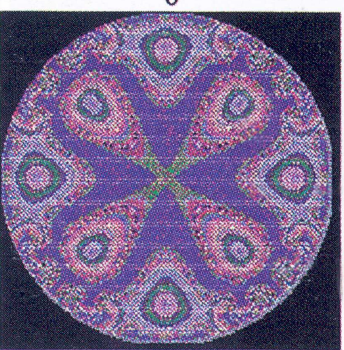

10

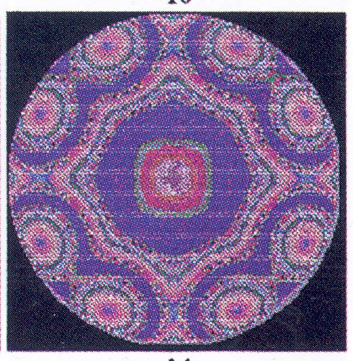

14

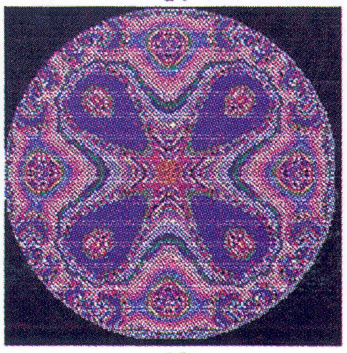

18

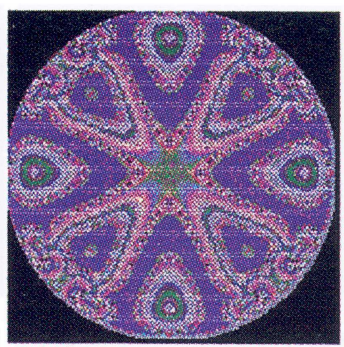

3

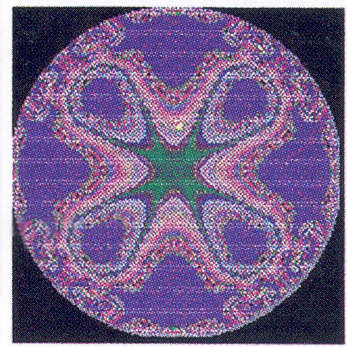

7

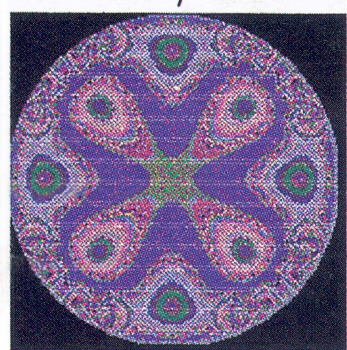

11

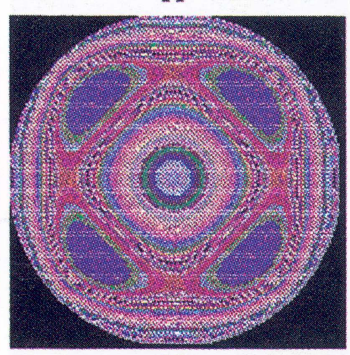

15

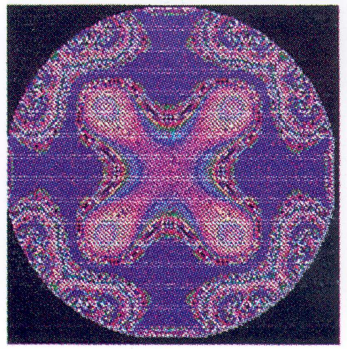

19
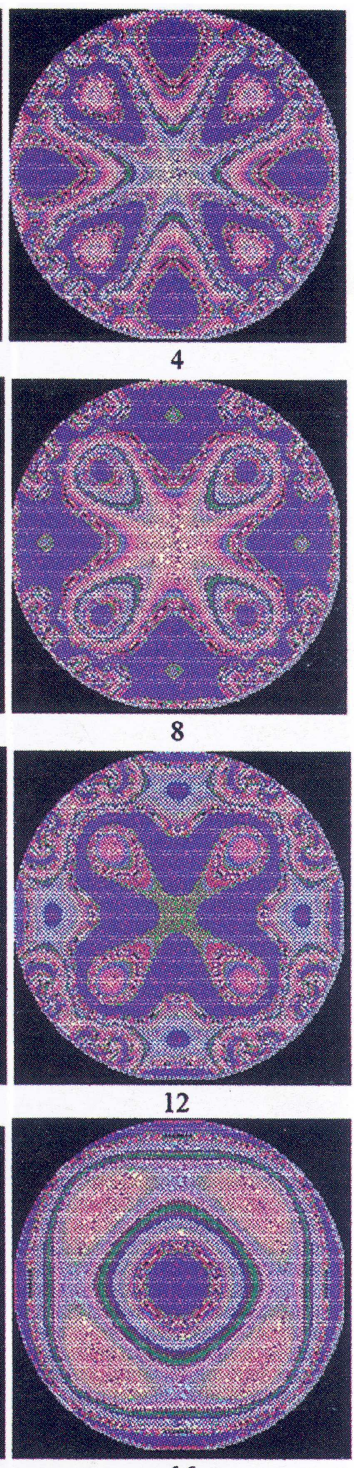

16

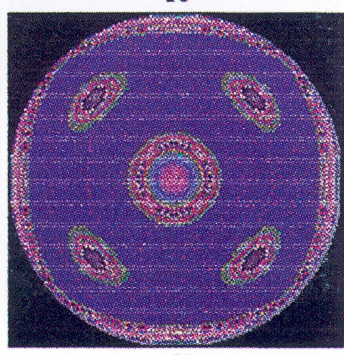

20

FIGURE 5 Mandalas calculated by the discrete chaotic dynamics algorithms. (See Color Plate IV.) 

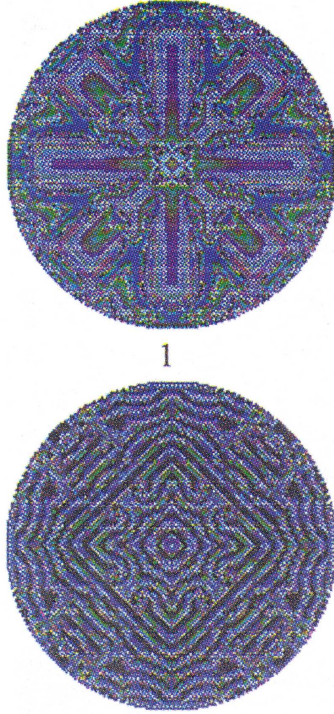

5

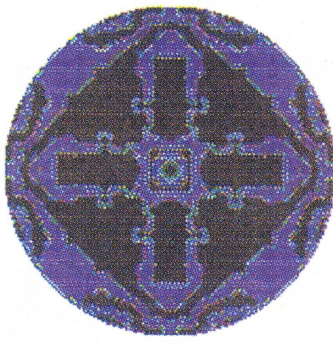

9

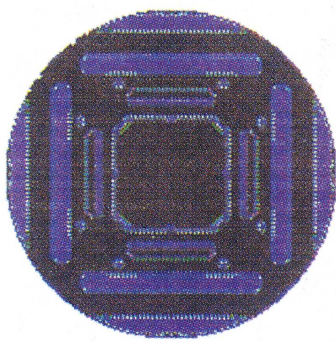

13

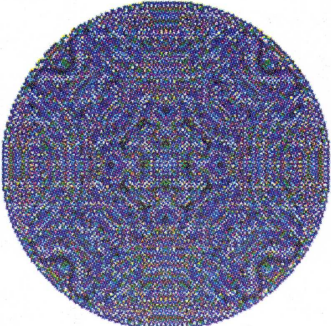

2

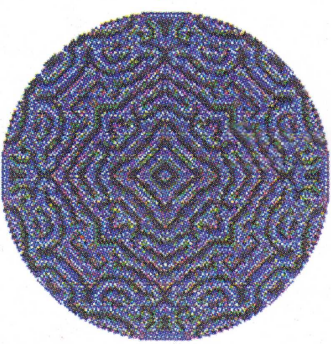

6

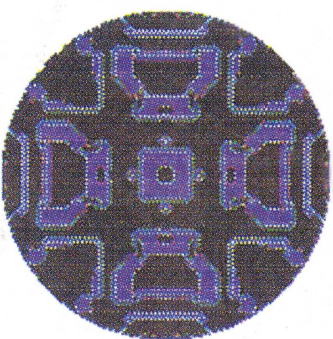

10

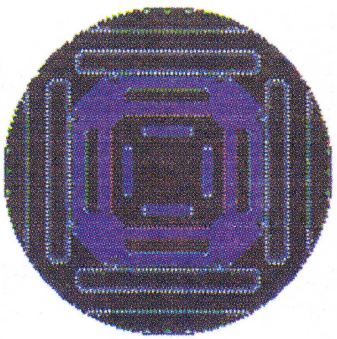

14

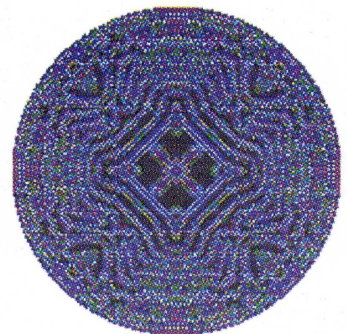

3

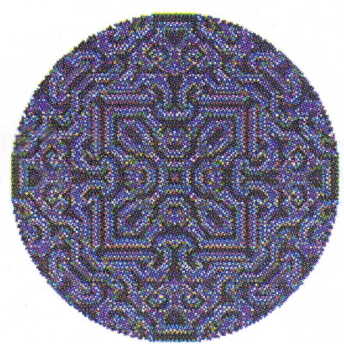

7

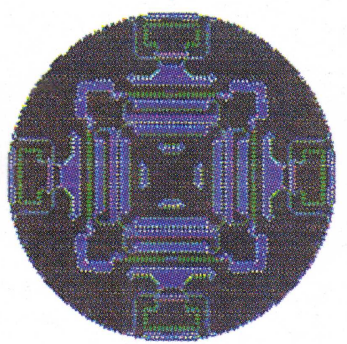

11

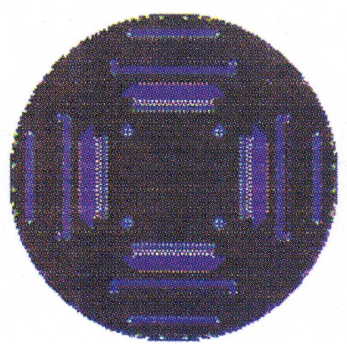

15

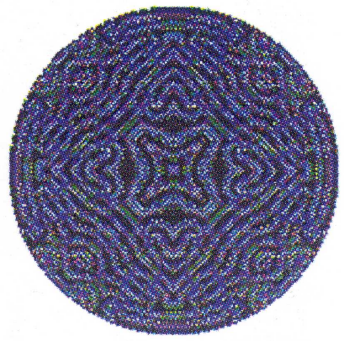

4

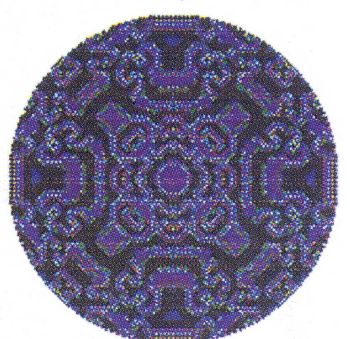

8

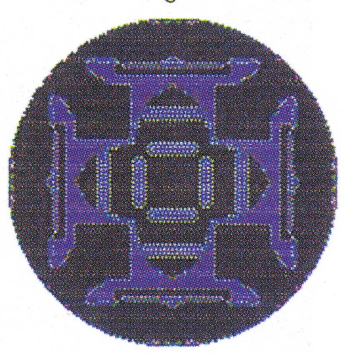

12

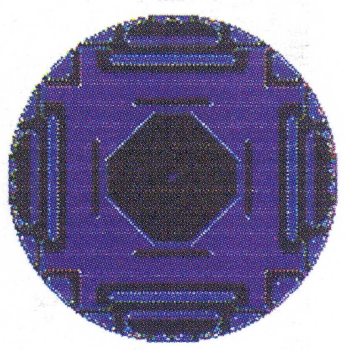

16

FIGURE 6 Mandalas calculated by the discrete chaotic dynamics algorithms. (See Color Plate V.)

high level of predictability. Hypothesis that distribution of the concentrations of chemicals in the brain neurons corresponds or somehow related to the brain creativity processes found some confirmation by generated patterns in a form of mandalas.

It seems natural to use our model for the creation of the "artificial brain" computer system based on the proposed mathematical model. In this way I do not see any constraints and taking into account computational simplicity of the discrete dynamics algorithms our "artificial brain" can be very efficient for solving different problems in the field of artificial intelligence.

\section{References}

[1] V. Gontar, "New theoretical approach for physicochemical reactions dynamics with chaotic behavior", in the book 
"Chaos in Chemistry and Biochemistry" World Scientific, London, 1993, pp. 225-247.

[2] C.G. Jung, Mandala Symbolism, Prinston University Press, 1972.

[3] L. Brand, " $\pi$-Theorem of the theory of dimentionality", Arch. Rational Mech. Anal., 1, 1957, 35-43.

[4] V. Gontar, "New principle and mathematical model for chemical reactions kinetics", R.J. Phys. Chem. 55(9), 1981, 2301-2304.
[5] V. Gontar, "Theoretical foundation for discrete dynamics of physicochemical systems. chaos, self-organization, time and space in complex system". Discrete Dynamics in Nature and Society, 1(1), 1997, 31-44. 


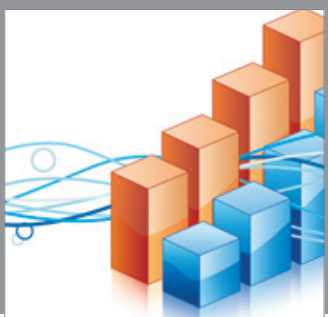

Advances in

Operations Research

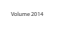

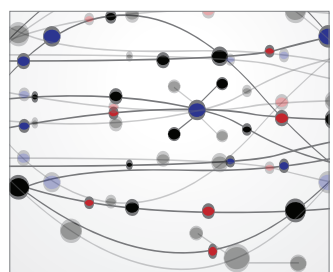

\section{The Scientific} World Journal
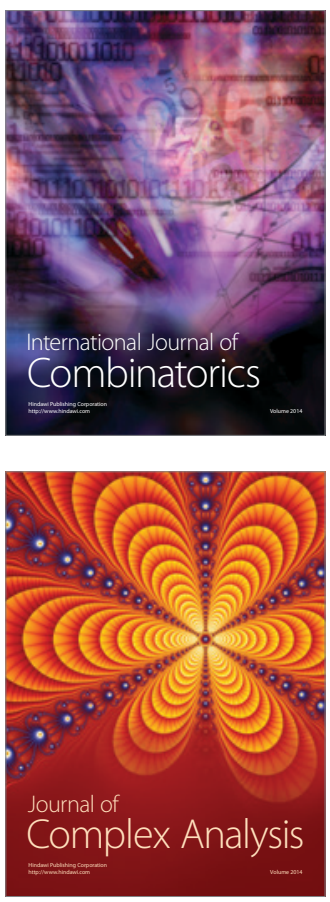

International Journal of

Mathematics and

Mathematical

Sciences
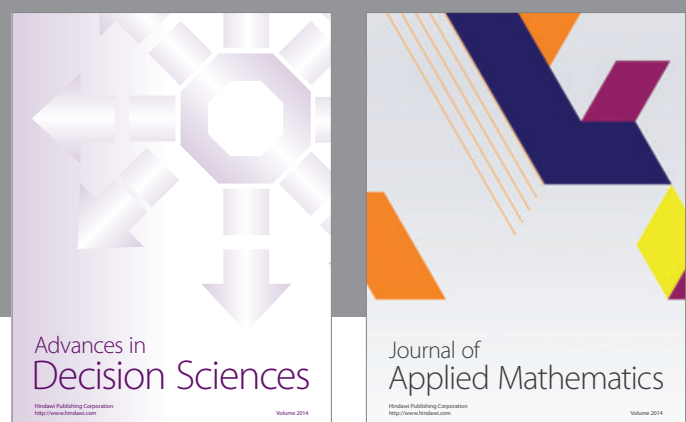

Journal of

Applied Mathematics
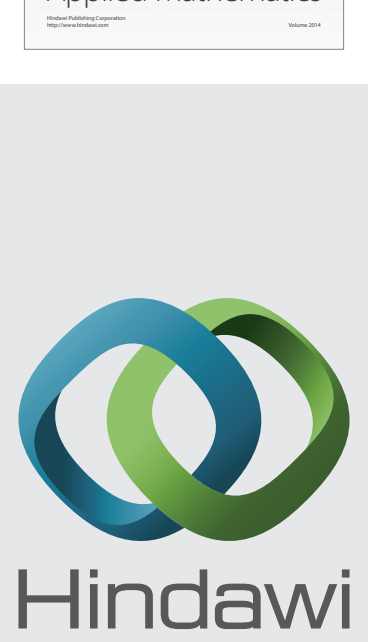

Submit your manuscripts at http://www.hindawi.com
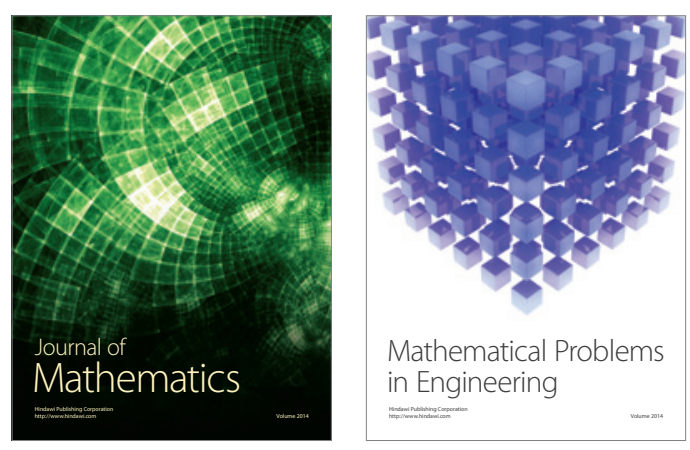

Mathematical Problems in Engineering
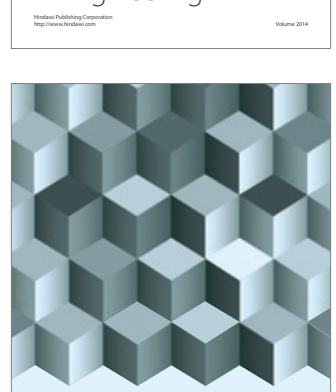

Journal of

Function Spaces
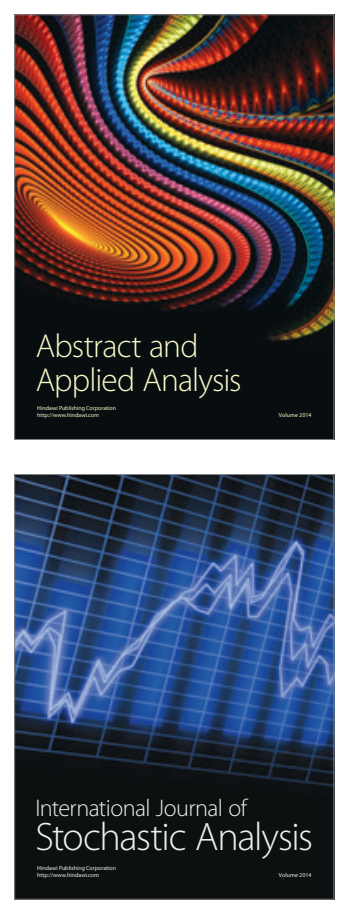

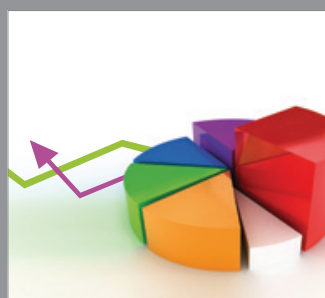

ournal of

Probability and Statistics

Promensencen
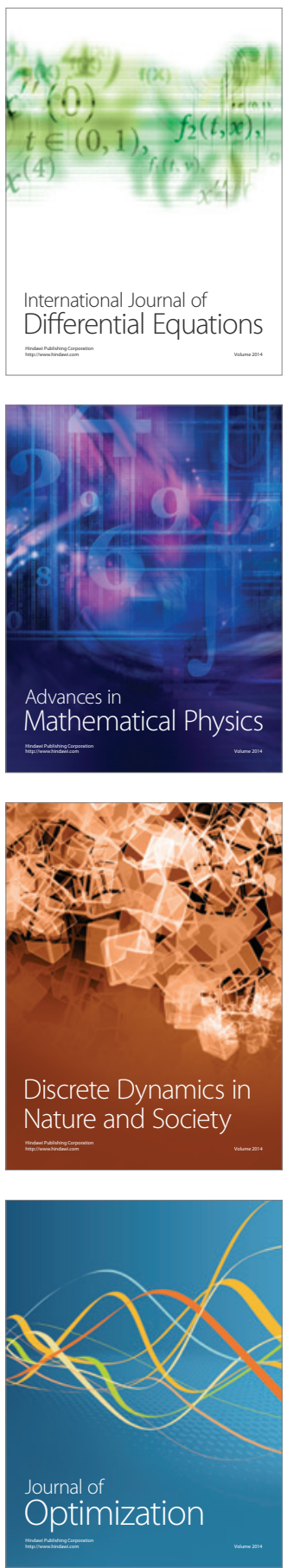